\title{
Increasing the Brightness of Cold Ion Beams by Suppressing Disorder-Induced Heating with Rydberg Blockade
}

\author{
D. Murphy, R. E. Scholten, ${ }^{*}$ and B. M. Sparkes \\ School of Physics, The University of Melbourne, Victoria 3010, Australia
}

(Received 27 July 2015; published 20 November 2015)

\begin{abstract}
A model for the equilibrium coupling of an ion system with varying initial hard-sphere Rydberg blockade correlations is used to quantify the suppression of disorder-induced heating in Coulombexpanding cold ion bunches. We show that bunches with experimentally achievable blockade parameters have an emittance reduced by a factor of 2.6 and increased focusability and brightness compared to a disordered bunch. Demonstrating suppression of disorder-induced heating is an important step in the development of techniques for the creation of beam sources with sufficient phase-space density for ultrafast, single-shot coherent diffractive imaging.
\end{abstract}

DOI: 10.1103/PhysRevLett.115.214802

PACS numbers: 29.27.Bd, 07.77.Ka, 37.10.-x, 52.27.Jt

Nanoscale material manipulation with focused ion beams [1], high resolution electron microscopy [2,3], and ultrafast and coherent electron diffraction imaging [4] require high flux low emittance sources of charged particles. The emittance, or focusability, of the beam determines important imaging capabilities such as the resolution and image acquisition time. Cold-atom electron and ion sources (CAEIS) [5,6] based on the photoionization of laser-cooled atomic gases are being developed for the production of low emittance beams, achieving source temperatures below $10 \mathrm{~K}$ for electrons [7] and $1 \mathrm{mK}$ for ions [8]. These sources produce charged particle bunches with arbitrarily shaped spatial profiles, short durations and high coherence [9-11], and high resolution focus suitable for ion microscopy [12].

Intrabeam Coulomb effects limit usable beam densities in applications that require short bunch duration, such as single-shot ultrafast electron diffraction where the expansion effects constrain the imaging capability of bunches with more than a few tens of particles [13]. At high beam densities, the nonlinear Coulomb expansion of bunches with nonuniform space-charge densities leads to loss of beam quality [14]. CAEIS ion bunches have been used as a platform for the study of space-charge effects, since low source temperature and high mass enable detailed measurements analogous to much shorter bunch durations appropriate to ultrafast electron beams [15]. The initial source distribution of CAEIS beams can be shaped to produce uniform charge distributions, which is predicted to alleviate space-charge induced emittance growth [14,16].

For beam sources with uniform charge distributions, the minimum achievable source temperature is ultimately limited by statistical Coulomb effects resulting from the discrete nature of the charged particles [17]. Particle beams cooled from initially high temperatures, such as electroncooled heavy ion beams in storage rings, encounter a cooling limit due to intrabeam scattering when operated at high beam density [18]. Bunches of initially cold charged particles created with uncorrelated initial positions, as from a CAEIS, have interparticle repulsive forces that are random in orientation and magnitude, leading to disorder-induced heating (DIH) $[19,20]$. DIH can, in principle, be suppressed by establishing spatial correlations in the initial particle distribution, for example, by ionization of a degenerate Fermi gas [21], or atoms in an optical lattice [22], or blockaded Rydberg-atom ensembles [23]. Simulations of the suppression of DIH in these precorrelated plasmas have been performed only in the absence of expansion, which, otherwise, prevents establishment of thermal equilibrium due to the effects of adiabatic cooling $[24,25]$. Models of DIH suppression through correlation in cold charged particle bunches, where rapid Coulomb expansion is unavoidable, are needed to establish limitations to beam quality on the basis of achievable experimental parameters.

In this Letter, we investigate the suppression of DIH in Coulomb-expanding cold ion beams with initial position correlations created through the ionization of a shaped blockaded Rydberg gas, where spatial correlations are introduced by interatomic interactions that prevent excitation of pairs of atoms that are in close proximity [26]. We model the DIH process using hard-sphere initial correlations $[27,28]$ to derive the equilibrium temperature and show, through detailed molecular-dynamics (MD) simulations, that the suppression of DIH calculated in equilibrium holds during the emittance nonconserving, nonequilibrium expansion process for uniform bunches. The effects of Coulomb interactions within an ion bunch are equivalent to those in an electron bunch, but occur on a longer time scale due to the larger ion mass [15]. Therefore, our model places an experimental limit on the reduction of emittance through hard-sphere correlations for any highdensity charged particle bunch, such as electrons used for single-shot, ultrafast diffraction studies. 
Emittance is a measure of the phase-space area occupied by a beam, and can be expressed in each spatial dimension $x$ as

$$
\epsilon=\frac{\sigma_{x}}{c} \sqrt{\frac{k_{B} T}{m}},
$$

where $\sigma_{x}$ is the root-mean-square (rms) beam size, $c$ the speed of light, $k_{B}$ the Boltzmann constant, $m$ the mass of one particle. The thermal energy is $k_{B} T=m \sigma_{v_{x}}^{2}\left(1-r_{x, v_{x}}^{2}\right)$ where $\sigma_{v_{x}}$ is the rms velocity spread and $r_{x, v_{x}}$ is the correlation coefficient of the $x$-axis position and velocity phase-space profile. At a beam waist, emittance is the product of the beam size and angular divergence, thereby determining the minimum focal size for a given focusing system. Achieving high beam brightness and coherence requires low beam emittance and, thus, low temperature. The internal structure and dynamics of a plasma can be described in terms of its temperature using the Coulomb coupling parameter $\Gamma$, which is the ratio of potential to thermal energy

$$
\Gamma=\frac{e^{2}}{4 \pi \varepsilon_{0} a k_{B} T},
$$

where $e$ is the elementary charge, $\varepsilon_{0}$ is the vacuum permittivity and the Wigner-Seitz radius $a=(3 / 4 \pi n)^{1 / 3}$ is the average interparticle separation for ion density $n$. For strong coupling $(\Gamma>1)$, the motion of individual particles is determined by the interparticle interactions rather than thermal diffusion.

For an initially disordered cold-ion plasma, $\Gamma$ may be high initially due to the low temperature, but DIH occurs with a characteristic time scale on the order of the inverse of the plasma frequency $\omega_{p}=\sqrt{n e^{2} / \varepsilon_{0} m}$, limiting the system to an equilibrium coupling parameter $\Gamma_{\mathrm{eq}} \sim 2$ in the absence of expansion [29]. At an ion density $n=10^{16} \mathrm{~m}^{-3}$, typical of a CAEIS using a magneto-optical trap (MOT) atom source, this implies rapid equilibration to a temperature $T_{c}=2 \mathrm{~K}, 5$ orders of magnitude higher than the temperature of the atoms before ionization.

Previous simulations have suggested that equilibrium coupling parameters as high as $\Gamma_{\mathrm{eq}}=35$, corresponding to much lower temperatures, may be reached in neutral nonexpanding plasmas produced through ionization of blockaded Rydberg atoms [23]. A pair of Rydberg atoms in a low angular momentum state with separation $R$ interact, to leading order, via the van der Waals (VDW) potential $V(R)=-C_{6} / R^{6}$. In an ensemble of atoms exposed to radiation coupling a ground state to a Rydberg level, with power-broadened linewidth $\nu$, any ground-state atom within the blockade radius $r_{b}=\left|C_{6} / \nu\right|^{1 / 6}$ of a Rydberg atom is prevented from being excited to the Rydberg level by the VDW interaction. For two rubidium atoms in the $60 \mathrm{~S}$ state that have $C_{6}=-140 \mathrm{GHz} \mu \mathrm{m}^{6}$ and linewidth $\nu=3 \mathrm{MHz}$, the blockade radius of $r_{b}=6 \mu \mathrm{m}$ [30] exceeds the mean interparticle separation $a$ of a random distribution at the typical ion density of $n=10^{16} \mathrm{~m}^{-3}$. Therefore, the blockade provides a mechanism for suppression of DIH and consequent reduction of the effective source temperature.

First, we consider the thermal equilibrium properties of a nonexpanding ion bunch with Rydberg blockade correlations. Following creation of a blockaded Rydberg ensemble created in a volume defined by a two-laser excitation process [9], our model assumes complete ionization of the Rydberg atoms by a switched electrostatic field in a time much less than the DIH time scale $\omega_{p}^{-1}$, which is achievable in existing MOT-based CAEIS accelerators [31]. The field accelerates the ions, and removes electrons from the system on a much shorter time scale than the ionic motion, such that they have a negligible effect on the ion temperature. Hard-sphere approximations for the VDW interaction have been shown to adequately approximate the spatial correlations [27] and excitation fractions [28] of high density blockaded Rydberg ensembles. The hardsphere approximation allows calculation of the internal energy of the ion configuration as a function of a dimensionless blockade parameter $r_{b} / a$, which we use to derive the equilibrium coupling parameter after DIH.

Using the approach for a disordered system [32], we calculate the equilibrium Coulomb coupling parameter $\Gamma_{\mathrm{eq}}$ of a nonexpanding ion system by, first, considering the conservation of energy per particle in the initial and final (equilibrium) ionic configurations. For potential energy, we use the internal binding energy per particle in a onecomponent plasma (OCP) system $u_{\mathrm{OCP}}$ [33]. In equilibrium, this is given by the OCP equation of state in the equilibrium strongly coupled fluid phase $\left(\Gamma_{\text {eq }} \lesssim 170\right)$ [34]

$u_{\mathrm{OCP}}=\frac{e^{2}}{4 \pi \varepsilon_{0} a}\left(-0.9+0.590673 \Gamma_{\mathrm{eq}}^{-2 / 3}-0.26569 \Gamma_{\mathrm{eq}}^{-1}\right)$.

For an initially disordered distribution, the initial internal binding energy $u_{i}=0$, as the ions have no structure and experience no initial confining force. Within one plasma period, the ions develop short-ranged OCP correlations with negative binding energy $u_{\mathrm{OCP}}$ balanced by a gain of kinetic energy. Conservation of energy for an initially cold, disordered distribution with a final thermal equilibrium potential $u_{f}=u_{\mathrm{OCP}}$ gives an equilibrium coupling parameter $\Gamma_{\mathrm{eq}}=2.23$ [17]. Ions with correlated initial positions have a nonzero initial binding energy so that less kinetic energy is gained per particle during thermal equilibration, leading to an increase in the Coulomb coupling parameter in the final state. For hard-sphere correlations, the PercusYevick (PY) equation gives a radial distribution function [35] that permits calculation of an analytical form of the internal energy under Coulomb interactions [36]

$$
u_{\mathrm{PY}}=-\frac{3 e^{2} \eta^{2 / 3}}{4 \pi \varepsilon_{0} a} \frac{1-\frac{1}{5} \eta+\frac{1}{10} \eta^{2}}{1+2 \eta},
$$




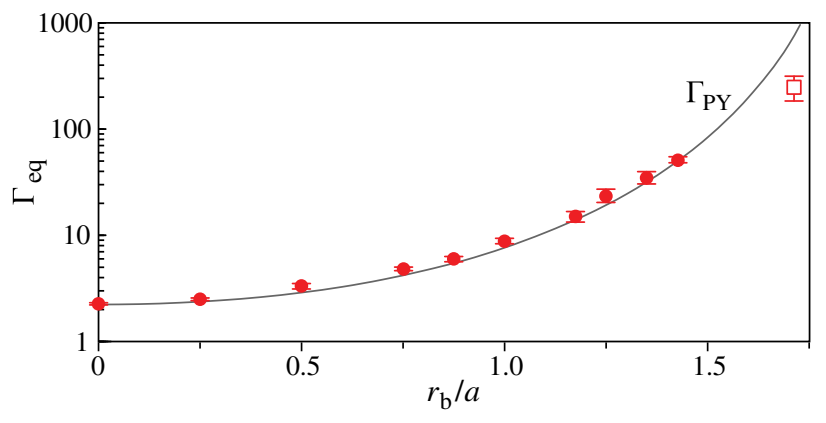

FIG. 1 (color online). Equilibrium Coulomb coupling parameter $\Gamma_{\text {eq }}$ of cold ion bunches with hard-sphere blockade parameter $r_{b} / a$ calculated using the Percus-Yevick hard-sphere distribution function $\Gamma_{\mathrm{PY}}$ (solid line) and by molecular dynamics simulation for configurations generated by random sequential addition (circles) and for a random-close-packed distribution at $r_{b} / a=$ 1.7 (square). Uncertainties in the MD data result from residual kinetic energy oscillations.

where $\eta=\frac{1}{8}\left(r_{b} / a\right)^{3}$ is the packing fraction of spheres of diameter $r_{b}$ in the hard-sphere approximation. Conservation of energy with $u_{i}=u_{\mathrm{PY}}$ then gives the PY hard-sphere equilibrium coupling parameter $\Gamma_{\mathrm{PY}}$ as a function of the blockade parameter $r_{b} / a$ (Fig. 1). For a blockade parameter of $r_{b} / a=1.2$ reported by experiments [28], $\Gamma_{\mathrm{PY}}=16$. The solution of $\Gamma_{\mathrm{PY}}$ for blockade parameters $r_{b} / a$ underpins the behavior of the coupling parameter observed in simulations of neutral plasmas with Rydberg blockade [23].

To quantify suppression of DIH in an expanding beam, we performed an $N$-body MD simulation of DIH in an infinite homogeneous system of ions with linear expansion by using periodic boundary conditions in a comoving cubic geometry with equations of motion from the direct Ewald summation method [37] (see Supplemental Material [38]). In the comoving coordinate frame, the effects of linear expansion, including adiabatic cooling, are described by scale factor $\alpha$ calculated for the free uniform Coulomb expansion of ions at an initial density $n^{\prime}$

$$
\frac{d^{2} \alpha}{d t^{2}}=\frac{n^{\prime} e^{2}}{3 \varepsilon_{0} m \alpha^{2}},
$$

where $\alpha=1$ immediately following ionization.

In experiments where ordering is established through blockade, for the same initial atomic density, the bunch charge will be smaller. In the presence of linear spacecharge expansion, higher density bunches expand more rapidly, so that brightness is independent of the bunch charge for a fixed source temperature [42]. Therefore, we simulate bunches with equal initial densities $n^{\prime}=1 \times$ $10^{16} \mathrm{~m}^{-3}$ for the ordered and disordered cases, with equal expansion rates, to allow for fair comparison. To compare the behavior of the $\mathrm{Rb}$ ion bunches that we simulate to other bunches of charged particles at different densities, including electrons, the time scales of the kinetic energy oscillations, heating and expansion scale as $\sqrt{m / n^{\prime}}$ and the emittance as $\tilde{\epsilon} \propto \sqrt{n^{\prime 1 / 3} / m}$.

$N=1000$ ions were generated in simulation by the random sequential addition of hard spheres in the periodic comoving cubic volume of side length $L=\left(N / n^{\prime}\right)^{1 / 3}$, which generated configurations with blockade parameters up to $r_{b} / a=1.43$, close to the saturation limit [43]. To illustrate the behavior of bunches with higher blockade parameters, we use a packing algorithm [44] to generate ion configurations close to the random hard-sphere close packing limit of $r_{b} / a \sim 1.72$. The ions were generated with zero initial temperature. Simulations showed no observable differences when repeated with initial temperatures of $100 \mu \mathrm{K}$, the typical temperature of atoms in a MOT. The comoving equations of motion were integrated through the Bulirsch-Stoer method with polynomial extrapolation [45]. Adaptive time steps were calculated to provide a constant error metric for the emittance. The scale factor $\alpha$, expansion rate $d \alpha / d t$, and the temperature of the ions were evaluated (see Supplemental Material [38]) to determine a dimensionless emittance

$$
\tilde{\epsilon} \equiv \frac{\alpha}{c} \sqrt{\frac{k_{B} T}{m}}
$$

such that a spherical ion bunch of initial rms size $\sigma_{x}{ }^{\prime}$ expanded to a size $\alpha \sigma_{x}{ }^{\prime}$ will have an emittance $\epsilon=\sigma_{x}{ }^{\prime} \tilde{\epsilon}$.

Simulations were initially performed in the absence of expansion (setting $d \alpha / d t=0$ ) to calculate the equilibrium coupling parameters $\Gamma_{\text {eq }}$ for varying blockade parameters $r_{b} / a$ in order to verify the PY prediction. The regular kinetic energy oscillations at twice the plasma frequency (seen in Fig. 2) result from the harmonic motion of ions about dynamic local potential minima. The equilibrium emittance and temperature decreases as the blockade parameter increases. The equilibrium coupling parameters for each simulation were found by averaging over plasma phases, which, for a nonexpanding bunch, is the product of plasma frequency and time, between $8 \pi$ and $16 \pi$. The MD simulations verify the PY OCP theory (Fig. 1) for distributions generated up to $r_{b} / a=1.43$ in nonexpanding thermal equilibrium.

Expanding ion bunches cool adiabatically, with decreasing plasma frequency for decreasing density as $\omega_{p} \propto \alpha^{-3 / 2}$. Asymptotically, the kinetic energy oscillations cease as the plasma phase $\varphi=\int_{0}^{t} \omega_{p} d t^{\prime}$ converges to $\varphi=1.2 \pi$ as $t \rightarrow \infty$, for the free Coulomb expansion (Eq. (5) of ions of any mass or initial density. Convergence of the plasma phase implies freezing of the ion motion in the expanding reference frame, so that we may expect the emittance to reach an equilibrium value, as adiabatic expansion cooling preserves bunch emittance, decreasing the temperature as $T \propto \alpha^{-2}$ [17]. Instead, we observe unbound emittance growth subsequent to the first temperature oscillation minimum at $\varphi=\pi$ (Fig. 3). The results, presented in terms of an effective source temperature $T^{\prime}=\alpha^{2} T$ of equivalentemittance noninteracting particles in the ion source region, 


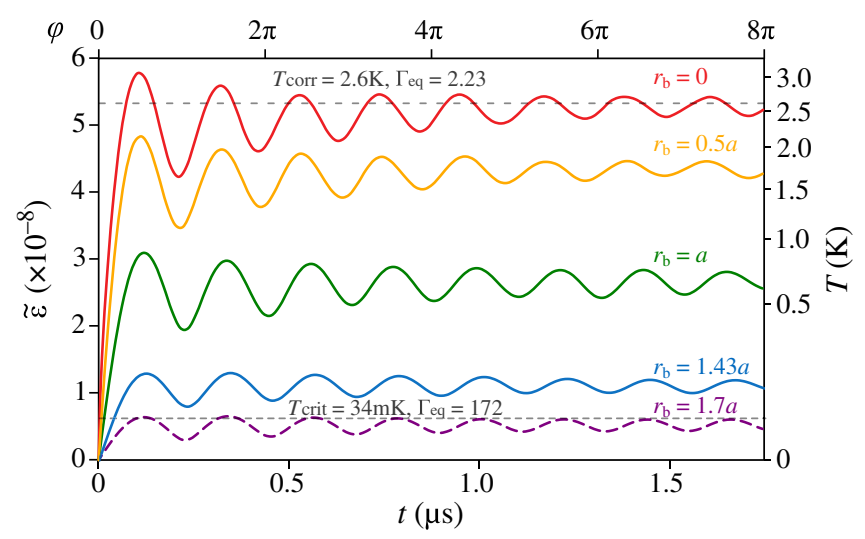

FIG. 2 (color online). Disorder-induced heating of nonexpanding ion bunches with hard-sphere initial correlations, labeled by the blockade parameter $r_{b} / a$. Emittance and temperature are calculated in time and in plasma phase $\varphi$, which is $2 \pi$ times the number of plasma periods after ionization, for ${ }^{85} \mathrm{Rb}$ ions at density $n^{\prime}=10^{16} \mathrm{~m}^{-3}$. Damped kinetic energy oscillations at twice the plasma frequency $\omega_{p}$ due to harmonic ion motion are observed. Hard-sphere Rydberg blockade reduces the equilibrium temperatures from the disordered $\left(r_{b}=0\right)$ correlation temperature $T_{\text {corr }}=2.6 \mathrm{~K}$, to near the crystallization phase boundary at $T_{\text {crit }}=34 \mathrm{mK}$.

show clear reduction in the magnitude of the emittance growth for blockaded initial distributions.

Modeling the harmonic ion motion about local potential minima in the expanding frame yields a nonadiabatic asymptotic temperature dependence of $T \propto \Gamma_{\text {eq }}^{-1} \alpha^{-1.8}$ (see Supplemental Material [38]). According to the harmonic oscillator description, the temperature dynamics during expansion are proportional to the predicted equilibrium coupling parameter $\Gamma_{\mathrm{PY}}$ for each blockade parameter. The reduction of the emittance, and the suppression of $\mathrm{DIH}$, can then be quantified as the ratio of the emittance of the blockaded system with equilibrium coupling $\Gamma_{\mathrm{eq}}=\Gamma_{\mathrm{PY}}\left(r_{b} / a\right)$ to the emittance of a disordered system which has $\Gamma_{\text {eq }}=2.23$

$$
\frac{\epsilon_{\text {blockade }}}{\epsilon_{\text {disorder }}}=\sqrt{\frac{2.23}{\Gamma_{\mathrm{PY}}\left(r_{b} / a\right)}} .
$$

The MD results of Fig. 4 verify that the suppression is unaffected by the expansion. The limit of the suppression depends on the extent of the blockade. For an ion beam created from a system with $r_{b} / a=1.2$ [28], we predict an equilibrium coupling parameter $\Gamma_{\mathrm{eq}}=16$, and an emittance lowered by a factor of 2.6. This emittance reduction would lead to a sevenfold increase in transverse brightness, which is proportional to $\epsilon^{-2}$. The minimum focal spot size $d$ would be 0.62 times the size with disorder, where $d \propto \epsilon^{1 / 2}$.

Suppression of DIH in cold ions would be a significant milestone in the advancement of charged particle beam

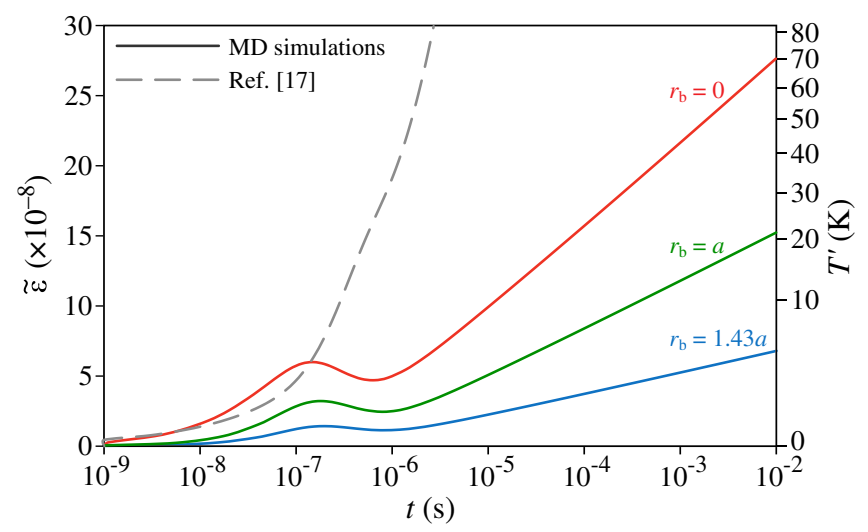

FIG. 3 (color online). Dimensionless emittance $\tilde{\epsilon}$ and effective temperature growth caused by disorder-induced heating in freely expanding ${ }^{85} \mathrm{Rb}^{+}$ion bunches at $10^{16} \mathrm{~m}^{-3}$ initial density, calculated by molecular-dynamics simulation. A model for the bunch temperature evolution [17] is found to overpredict the emittance growth at times beyond the first oscillation maxima. The presence of Rydberg blockade, labeled by the blockade radius in terms of the Wigner-Seitz radius, increases the initial ion correlations thereby reducing the heating.

source technology, as the DIH effect, otherwise, presents a limitation to beam focusability and brightness. Ultimately, single-shot ultrafast electron diffraction may require electron beams of a phase-space density close to the quantum degeneracy limit [46], which will not be possible unless DIH is negligible. For ions, creating bunches with higher blockade parameters and further reduced emittance may be possible through precise variation of the laser frequency and intensity during the Rydberg excitation process [47], Rydberg excitation of atoms in an optical lattice with high filling fraction $[22,48]$, or ionization of atoms in spatially tailored microtrap arrays [49].

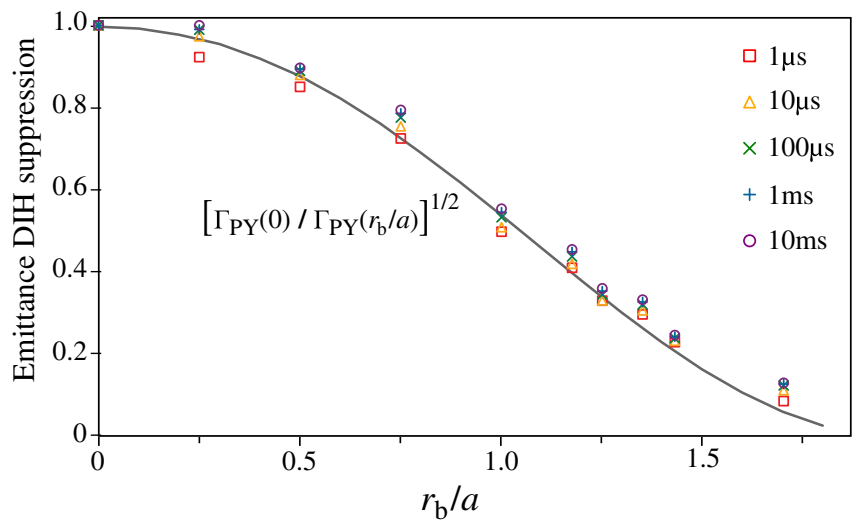

FIG. 4 (color online). Suppression of disorder-induced heating, expressed as the ratio of blockaded to disordered emittance for different blockade parameters at different expansion times. The suppression remains constant throughout the linear Coulomb expansion process, as predicted with the hard-sphere model for the thermal equilibrium coupling parameter (solid line). 
In conclusion, the results presented here quantitatively predict the extent to which disorder-induced emittance growth can be suppressed using Rydberg blockade, and guide further development of high-brightness cold charged particle beams for applications including ultrafast electron diffraction.

B. M.S. gratefully acknowledges the support of a University of Melbourne McKenzie Fellowship. This work was supported by the Australian Research Council Discovery Project No. DP140102102.

*scholten@unimelb.edu.au

[1] J. Orloff, High-resolution focused ion beams, Rev. Sci. Instrum. 64, 1105 (1993).

[2] T. LaGrange, M. R. Armstrong, K. Boyden, C. G. Brown et al., Single-shot dynamic transmission electron microscopy, Appl. Phys. Lett. 89, 044105 (2006).

[3] K. W. Urban, Studying Atomic Structures by AberrationCorrected Transmission Electron Microscopy, Science 321, 506 (2008).

[4] J. C. Williamson, J. Cao, H. Ihee, H. Frey, and A. H. Zewail, Clocking transient chemical changes by ultrafast electron diffraction, Nature (London) 386, 159 (1997).

[5] B. J. Claessens, S. B. van der Geer, G. Taban, E. J. D. Vredenbregt, and O.J. Luiten, Ultracold Electron Source, Phys. Rev. Lett. 95, 164801 (2005).

[6] J. L. Hanssen, E. A. Dakin, J. J. McClelland, and M. Jacka, Using laser-cooled atoms as a focused ion beam source, J. Vac. Sci. Technol. B 24, 2907 (2006).

[7] W. J. Engelen, E. P. Smakman, D. J. Bakker, O. J. Luiten, and E. J. D. Vredenbregt, Effective temperature of an ultracold electron source based on near-threshold photoionization, Ultramicroscopy 136, 73 (2014).

[8] N. Debernardi, M. P. Reijnders, W. J. Engelen, T. T. J. Clevis, P. H. A. Mutsaers, O. J. Luiten, and E. J. D. Vredenbregt, Measurement of the temperature of an ultracold ion source using time-dependent electric fields, J. Appl. Phys. 110, 024501 (2011).

[9] A. J. McCulloch, D. V. Sheludko, S. D. Saliba, S. C. Bell, M. Junker, K. A. Nugent, and R. E. Scholten, Arbitrarily shaped high-coherence electron bunches from cold atoms, Nat. Phys. 7, 785 (2011).

[10] A. J. McCulloch, D. V. Sheludko, M. Junker, and R. E. Scholten, High-coherence picosecond electron bunches from cold atoms, Nat. Commun. 4, 1692 (2013).

[11] M. W. van Mourik, W. J. Engelen, E. J. D. Vredenbregt, and O. J. Luiten, Ultrafast electron diffraction using an ultracold source, Struct. Dyn. 1, 034302 (2014).

[12] K. A. Twedt, L. Chen, and J. J. McClelland, Scanning ion microscopy with low energy lithium ions, Ultramicroscopy 142, 24 (2014).

[13] F. O. Kirchner, A. Gliserin, F. Krausz, and P. Baum, Laser streaking of free electrons at $25 \mathrm{keV}$, Nat. Photonics 8, 52 (2014).

[14] O. J. Luiten, S. B. Van der Geer, M. J. De Loos, F. B. Kiewiet, and M. J. van der Wiel, How to Realize Uniform
Three-Dimensional Ellipsoidal Electron Bunches, Phys. Rev. Lett. 93, 094802 (2004).

[15] D. Murphy, R. W. Speirs, C. T. Putkunz, A. J. McCulloch, B. M. Sparkes, and R.E. Scholten, Detailed observation of space-charge dynamics using ultracold ion bunches, Nat. Commun. 5, 4489 (2014).

[16] P. Musumeci, J. T. Moody, R. J. England, J. B. Rosenzweig, and T. Tran, Experimental Generation and Characterization of Uniformly Filled Ellipsoidal Electron-Beam Distributions, Phys. Rev. Lett. 100, 244801 (2008).

[17] J. M. Maxson, I. V. Bazarov, W. Wan, H. A. Padmore, and C. E. Coleman-Smith, Fundamental photoemission brightness limit from disorder induced heating, New J. Phys. 15, 103024 (2013).

[18] M. Steck, K. Beckert, H. Eickhoff, B. Franzke, F. Nolden, H. Reich, B. Schlitt, and T. Winkler, Anomalous Temperature Reduction of Electron-Cooled Heavy Ion Beams in the Storage Ring ESR, Phys. Rev. Lett. 77, 3803 (1996).

[19] D. O. Gericke and M. S. Murillo, Disorder-induced heating of ultracold plasmas, Contrib. Plasma Phys. 43, 298 (2003).

[20] G. ten Haaf, S. H. W. Wouters, S. B. van der Geer, E. J. D. Vredenbregt, and P.H. A. Mutsaers, Performance predictions of a focused ion beam from a laser cooled and compressed atomic beam, J. Appl. Phys. 116, 244301 (2014).

[21] M. S. Murillo, Using Fermi Statistics to Create Strongly Coupled Ion Plasmas in Atom Traps, Phys. Rev. Lett. 87, 115003 (2001).

[22] T. Pohl, T. Pattard, and J. M. Rost, On the possibility of 'correlation cooling' of ultracold neutral plasmas, J. Phys. B 37, L183 (2004).

[23] G. Bannasch, T. C. Killian, and T. Pohl, Strongly Coupled Plasmas via Rydberg Blockade of Cold Atoms, Phys. Rev. Lett. 110, 253003 (2013).

[24] T. Pohl, T. Pattard, and J. M. Rost, Relaxation to Nonequilibrium in Expanding Ultracold Neutral Plasmas, Phys. Rev. Lett. 94, 205003 (2005).

[25] P. McQuillen, T. Strickler, T. Langin, and T. C. Killian, Ion temperature evolution in an ultracold neutral plasma, Phys. Plasmas 22, 033513 (2015).

[26] E. Urban, T. A. Johnson, T. Henage, L. Isenhower, D. D. Yavuz, T. G. Walker, and M. Saffman, Observation of Rydberg blockade between two atoms, Nat. Phys. 5, 110 (2009).

[27] D. Petrosyan, M. Höning, and M. Fleischhauer, Spatial correlations of Rydberg excitations in optically driven atomic ensembles, Phys. Rev. A 87, 053414 (2013).

[28] M. Robert-de-Saint-Vincent, C. S. Hofmann, H. Schempp, G. Günter, S. Whitlock, and M. Weidemüller, Spontaneous Avalanche Ionization of a Strongly Blockaded Rydberg Gas, Phys. Rev. Lett. 110, 045004 (2013).

[29] S. D. Bergeson, A. Denning, M. Lyon, and F. Robicheaux, Density and temperature scaling of disorder-induced heating in ultracold plasmas, Phys. Rev. A 83, 023409 (2011).

[30] J. D. Pritchard, D. Maxwell, A. Gauguet, K. J. Weatherill, M. P. A. Jones, and C. S. Adams, Cooperative Atom-Light Interaction in a Blockaded Rydberg Ensemble, Phys. Rev. Lett. 105, 193603 (2010). 
[31] G. Taban, M. P. Reijnders, S. C. Bell, S. B. van der Geer, O. J. Luiten, and E. J. D. Vredenbregt, Design and validation of an accelerator for an ultracold electron source, Phys. Rev. ST Accel. Beams 11, 050102 (2008).

[32] M. Lyon, S. D. Bergeson, and M. S. Murillo, Limit of strong ion coupling due to electron shielding, Phys. Rev. E 87, 033101 (2013).

[33] G. Zwicknagel, Molecular Dynamics Simulations of the Dynamics of Correlations and Relaxation in an OCP, Contrib. Plasma Phys. 39, 155 (1999).

[34] D. H. E. Dubin and T. M. Oneil, Trapped nonneutral plasmas, liquids, and crystals (the thermal equilibrium states), Rev. Mod. Phys. 71, 87 (1999).

[35] G. Stell, The Percus-Yevick equation for the radial distribution function of a fluid, Physica (Utrecht) 29, 517 (1963).

[36] H. E. DeWitt and Y. Rosenfeld, Derivation of the one component plasma fluid equation of state in strong coupling, Phys. Lett. A 75, 79 (1979).

[37] A. Y. Toukmaji and J. A. Board, Jr., Ewald summation techniques in perspective: a survey, Comput. Phys. Commun. 95, 73 (1996).

[38] See Supplemental Material at http://link.aps.org/ supplemental/10.1103/PhysRevLett.115.214802 for derivation of the equations of motion, and for details of the evaluation of ion temperature in the simulations and the asymptotic temperature dependence of the expanding ions, which contains Refs. [39-41].

[39] C. Kittel and P. McEuen, Introduction to Solid State Physics (Wiley, New York, 1986).

[40] M. J. L. Sangster and M. Dixon, Interionic potentials in alkali halides and their use in simulations of the molten salts, Adv. Phys. 25, 247 (1976).
[41] R. T. Farouki and S. Hamaguchi, Thermodynamics of strongly-coupled Yukawa systems near the one-componentplasma limit. II. Molecular dynamics simulations, J. Chem. Phys. 101, 9885 (1994).

[42] O. J. Luiten, Beyond the RF Photogun in The Physics and Applications of High Brightness Electron Beams: Proceedings of the ICFA Workshop, Chia Laguna, Sardinia, 2002, Vol. 1 (World Scientific, Singapore, 2003), pp. 108-126.

[43] S. Torquato, O. U. Uche, and F. H. Stillinger, Random sequential addition of hard spheres in high Euclidean dimensions, Phys. Rev. E 74, 061308 (2006).

[44] W. S. Jodrey and E. M. Tory, Computer simulation of close random packing of equal spheres, Phys. Rev. A 32, 2347 (1985).

[45] W. H. Press, B. P. Flannery, S. A. Teukolsky, and W. T. Vetterling, Numerical Recipes in C (Cambridge University Press, Cambridge, England, 1992).

[46] B. W. Reed, M. R. Armstrong, N. D. Browning, G. H. Campbell, J. E. Evans, T. LaGrange, and D. J. Masiel, The Evolution of Ultrafast Electron Microscope Instrumentation, Microsc. Microanal. 15, 272 (2009).

[47] T. Pohl, E. Demler, and M. D. Lukin, Dynamical Crystallization in the Dipole Blockade of Ultracold Atoms, Phys. Rev. Lett. 104, 043002 (2010).

[48] J. Schachenmayer, I. Lesanovsky, A. Micheli, and A. J. Daley, Dynamical crystal creation with polar molecules or Rydberg atoms in optical lattices, New J. Phys. 12, 103044 (2010).

[49] F. Nogrette, H Labuhn, S. Ravets, D. Barredo, L. Béguin, A. Vernier, T. Lahaye, and A. Browaeys, Single-Atom Trapping in Holographic 2D Arrays of Microtraps with Arbitrary Geometries, Phys. Rev. X 4, 021034 (2014). 


\section{University Library}

\section{- M M N E R VA A gateway to Melbourne's research publications}

Minerva Access is the Institutional Repository of The University of Melbourne

Author/s:

Murphy, D;Scholten, RE;Sparkes, BM

Title:

Increasing the Brightness of Cold lon Beams by Suppressing Disorder-Induced Heating with Rydberg Blockade

Date:

2015-11-20

Citation:

Murphy, D., Scholten, R. E. \& Sparkes, B. M. (2015). Increasing the Brightness of Cold Ion Beams by Suppressing Disorder-Induced Heating with Rydberg Blockade. PHYSICAL REVIEW LETTERS, 115 (21), https://doi.org/10.1103/PhysRevLett.115.214802.

Persistent Link:

http://hdl.handle.net/11343/116473 